\title{
Representation of Universal Planar Automata by Autonomous Input Signals
}

\section{A. Molchanov}

Saratov State University, Russia, 410012, Saratov, Astrahanskaya st., 83, v.molchanov @inbox.ru

Universal planar automata are universally attracted objects in the category of automata, whose sets of states and output signals are endowed with structures of planes. The main result of the paper shows that any universal planar automaton is isomorphic to a many-sorted algebraic system canonically constructed from autonomous input signals of the automaton.

Key words: automata, semigroups, planes, many-sorted algebraic systems.

\section{References}

1. Plotkin B. I., Greenglaz L. Ja., Gvaramija A. A. Algebraic structures in automata and databases theory. Singapore, River Edge, NJ, World Scientific, 1992. (Rus. ed.: Plotkin B. I., Gringlaz L. Ia., Gvaramiia A. A. Elementy algebraicheskoi teorii avtomatov. Moscow, Vysshaia shkola, 1994, 192 p.)

2. Simovici Dan A. On the theory of reduction of semilatticial automata. An. Sti. ale Univ. «Al. l. Cuza»Din Iasi. (Ser. Nouă). Sec. 1a., 1976, vol. 22, no. 1, pp. 107110.

3. Gécseg F. O proizvedeniiakh uporiadochennykh avtomatov. I [On products of ordered automata. I]. Acta Sci. Math., 1963, vol. 24, no. 3-4, pp. 244-250 (in Russian)

4. Gécseg F. O proizvedeniah uporadochennyh avtomatov. II [On products of ordered automata. II]. Acta Sci. Math., 1964, vol. 25, no. 1-2, pp. 124-128 (in Russian).

5. Eilenberg S. Automata, languages and machines. Vol. B. New York, San Francisco, London, Academic Press, 1976, $451 \mathrm{p}$.

6. Introduction to finite geometries. Amsterdam, NorthHolland Publishing Co., 1976. (Russ. ed.: Kartesi F. Vvedenie v konechnye geometrii. Moscow, Nauka, 1980, 320 p.)

7. Ulam S. M. A Collection of Mathematical Problems. New Mexico, Los Alamos Scientific Laboratories, 1960. (Rus. ed.: Ulam S. Nereshennye matematicheskie zadachi. Moscow, Nauka, 1964, 168 p.)

8. Gluskin L. M. Polugruppy i kol'tsa endomorfizmov lineinykh prostranstv [Semigroups and rings of endomorphisms of linear spaces]. Izv. Akad. Nauk SSSR Ser. Mat., 1959, vol. 23, pp. 841-870 (in Russian).

9. Jonson B. Topics in Universal Algebras. Lecture Notes in Mathematics. Berlin, Heidelberg, New York, Springer Verlag, 1972, 220 p.

10. Konig D. Theorie der endlichen und unendlichen Graphen. Leipzig, Acad. Verlag M. B. H., 1936, 258 p.

11. Krasner M. Endotheorie de Galois abstraita. Semin. Dubriel, Dubriel-Jacotin, Lesieur et Pisot. Fac. sci. Paris, 1968-1969(1970), vol. 22, no 1, pp. 6/01-6/19.

12. Molchanov V. A. A universal planar automaton is determined by its semigroup of input symbols. Semigroup Forum, 2011, vol. 82, pp. 1-9.

13. Molchanov V. A. Konkretnaia kharakteristika universal'nykh planarnykh avtomatov [On concrete characterization of universal planar automata]. Matematika. Mehanika [Mathematics. Mechanics]. Saratov, Saratov Univ. Press, 2011, iss. 13, pp. 67-69 (in Russian).

14. Molchanov V. A. On relatively elementary definability of the class of universal planar automata in the class of all semigroups. Algebra i matematicheskaia logika: tez. dokl. Mezhdunar. konf., posviashch. 100-letiiu so dnia rozhdeniia $V$. $V$. Morozova [Proc. of the international conference dedicated to 100-th anniversary of V. V. Morozov and youth school-conf. «Modern Problems of Algebra \& Mathematical Logic»]. Kazan, 2011, pp. 145-147 (in Russian).

15. Ershov Yu. L. Problemy razreshimosti $i$ konstruktionye modeli [Problems of decidability and constructive models]. Moscow, Nauka, 1980, 416 p. (in Russian).

УДК 519.83

\section{МОДЕЛИ МНОГОКРИТЕРИАЛЬНОЙ ОПТИМИЗАЦИИ ПО КАЧЕСТВЕННЫМ КРИТЕРИЯМ}

\section{В. В. Розен ${ }^{1}$, Д. С. Смирнова ${ }^{2}$}

\begin{abstract}
${ }^{1}$ Доктор фризико-математических наук, заведующий касредрой геометрии, Саратовский государственный университет им. Н. Г. Чернышевского, Rozenvv@ info.sgu.ru

${ }^{2}$ Аспирантка касредры геометрии, Саратовский государственный университет им. Н. Г. Чернышевского, smirnova@okeankv.ru

Рассматриваются математические модели принятия решений по многим качественным критериям. Основная задача состоит в построении отношения предпочтения на множестве допустимых альтернатив и исследовании его математических свойств. Предложено два метода сужения паретовского оптимума: задание отношения частичного порядка на множестве критериев и выделение важнейших групп критериев.
\end{abstract}

Ключевые слова: модель многокритериальной оптимизации, отношение предпочтения, оптимальность по Парето, выигрывающая коалиция критериев. 


\section{ВВЕДЕНИЕ}

Первичная информация, относящаяся к данным многокритериальной задачи принятия решения (ЗПР), может быть представлена в виде таблицы, строки которой соответствуют изучаемым объектам, а столбцы - признакам, характеризующим эти объекты. В логике выделяют различные типы признаков в зависимости от того, какова структура соотношений между значениями признака. В первом приближении признаки объектов можно разделить на количественные и качественные. Разница между ними состоит в том, что значение количественного признака задается числом (которое является результатом измерения признака в некоторой числовой шкале), в то время как значения качественного признака связаны с естественным упорядочением по степени проявления этого признака. Необходимо отметить, что современная теория многокритериальной оптимизации, фактически, ограничивается количественными признаками, сводя тем самым качество к количеству и игнорируя при этом специфику качественных признаков. Между тем, именно качественные признаки характеризуют многие важные стороны изучаемых объектов и явлений; например, в экономике качественными признаками продукции являются такие признаки, как «удобство», «эргономичность», «эстетичность», «модность» и т. п.

Данная работа посвящена общим вопросам построения и исследования математических моделей многокритериальных ЗПР с качественными критериями. При построении математической модели задачи многокритериальной оптимизации необходимо задать множество допycmuмьх альтернатив (в качестве которых в конкретных ЗПР выступают интересующие нас объекты, а также варианты действий, планы, программы и т. п.), и критерии для оценки этих альтернатив. Заметим, что критериями могут служить как естественные, так и искусственные признаки (показатели, свойства), а также различные их комбинации. Формально математическая модель задачи многокритериальной оптимизации может быть представлена в виде набора

$$
G=\left\langle A,\left(q_{j}\right)_{j \in J}\right\rangle
$$

где А - непустое множество допустимых альтернатив, $\left(q_{j}\right)_{j \in J}$ критерии оценки этих альтернатив. Качественный критерий $q_{j}$ (локальный критерий качества) характеризуется тем, что шкалой для его измерения служит некоторое линейно упорядоченное множество (цепь) $\left\langle C_{j}, \leq^{j}\right\rangle$; формально $q_{j}$ представляет собой отображение $q_{j}: A \rightarrow C_{j}$. Каждой альтернативе $a \in A$ сопоставляется вектор $q(a)=\left(q_{j}(a)\right)_{j \in J}$, называемый векторной оценкой альтернативы $а$ и содержащий всю информацию об этой альтернативе; при этом в теоретическом анализе сравнение альтернатив заменяется сравнением их векторных оценок.

Иногда на отображение $q: A \rightarrow \prod_{j \in J} C_{j}$ накладывают дополнительное условие:

$$
(\forall j \in J) q_{j}\left(a_{1}\right)=q_{j}\left(a_{2}\right) \Rightarrow a_{1}=a_{2} .
$$

Обозначим через $K$ класс моделей многокритериальной оптимизации вида (1) с дополнительным условием (2).

Одной из важнейших задач многокритериальной оптимизации является построение отношения предпочтения на множестве альтернатив; решение этой задачи предполагает задание некоторого решающего правила. Наиболее известным решающим правилом является Парето-предпочтение $\omega^{\text {Par }}$, которое для модели (1) с качественными критериями принимает вид

$$
a_{1} \leq^{\omega^{\text {Par }}} a_{2} \Leftrightarrow(\forall j \in J) q_{j}\left(a_{1}\right) \leq^{j} q_{j}\left(a_{2}\right) .
$$

Парето-предпочтение является отношением порядка (или квазипорядка) на множестве альтернатив, и альтернативы, максимальные по этому отношению, называются эффективными или оптимальными по Парето, а множество этих альтернатив составляет паретовский оптимум. Ключевой принцип многокритериальной оптимизации может быть сформулирован в виде тезиса: выбор оптимальной альтернативы должен производиться из паретовского оптимума [1]. Этот принцип, фактически, дает необходимые условия оптимального решения в многокритериальной ЗПР. К сожалению, в типичных случаях паретовский оптимум оказывается достаточно «обширным», поэтому возникает 
сложная - как в принципиальном, так и в техническом отношении - проблема его сужения (в идеале - до одного элемента). Основное содержание настоящей работы связано с разработкой методов сужения паретовского оптимума для задач многокритериальной оптимизации с качественными критериями.

Отметим, что любые две альтернативы, принадлежащие паретовскому оптимуму, являются несравнимыми по Парето, поэтому выбор одной из двух предъявленных Парето-оптимальных альтернатив всегда является компромиссом: улучшая один из критериев, мы обязательно ухудшаем, хотя бы один из других. В этом заключается сложность логического анализа проблемы сужения паретовского оптимума. Все методы, направленные на решение этой проблемы, основаны на некоторой дополнительной информации о соотношении локальных критериев между собой или о свойствах оптимального решения. Предлагаемый нами подход сужения паретовского оптимума схематически может быть представлен в следующем виде. На основе дополнительной информации об относительной важности локальных критериев строится некоторое отношение предпочтения $\rho$ на множестве альтернатив, содержащее Парето-предпочтение $\omega^{P a r}$; это отношение позволяет сравнивать по предпочтению некоторые альтернативы, не сравнимые по Парето. Включение $\rho \supseteq \omega^{P a r}$ влечет обратное включение для множеств максимальных элементов относительно соответствующих предпочтений: $M_{\rho} \subseteq M_{\omega \text { Par, }}$ т. е. множество альтернатив, максимальных относительно предпочтения $\rho$, будет некоторым сужением паретовского оптимума. В работе рассмотрены два конкретных метода сужения паретовского оптимума; один из них основан на частичном упорядочении локальных критериев по их относительной важности, а другой - на выделении важнейших групп критериев.

\section{1. ЧАСТИЧНОЕ УПОРЯДОЧЕНИЕ КРИТЕРИЕВ ПО ВАЖНОСТИ}

Предположим, имеется дополнительная информация об относительной важности локальных критериев в форме отношения строгого частичного порядка «<» на множестве $J$; соотношение $i<j$ означает, что критерий $i$ является более важным, чем критерий $j$. Определим на множестве альтернатив модели (1) отношение предпочтения $\omega$ формулой

$$
a_{1} \leq^{\omega} a_{2} \Leftrightarrow(\forall j \in J)\left(q_{j}\left(a_{1}\right) \leq^{j} q_{j}\left(a_{2}\right) \vee(\exists i<j) q_{i}\left(a_{1}\right)<^{i} q_{i}\left(a_{2}\right)\right) .
$$

Всегда выполнено включение $\omega \supseteq \omega^{\text {Par }}$, которое влечет сужение паретовского оптимума. В работе [2] установлен следующий результат.

Теорема 1. Для того чтобы для любой модели $G \in K$ отношение $\omega$ являлось отношением порядка на множестве $A$, необходимо и достаточно чтобы упорядоченное множество $(J,<)$ удовлетворяло условию обрыва убывающих цепей (условию ОУЦ).

Отметим два крайних случая этой конструкции: (а) отношение < является пустым; и (б) отношение «<» является линейным порядком. В случае (а) отношение предпочтения $\omega$ совпадает с Парето-предпочтением, определенным формулой (3), а в случае (б) оно является лексикографическим упорядочением. В последнем случае паретовский оптимум сокращается до единственного элемента, который и считается оптимальным решением задачи многокритериальной оптимизации. Оба отмеченных случая являются крайними и на практике встречаются весьма редко. Типичным для практики является случай, когда имеется частичное упорядочение критериев по относительной важности.

Для моделей многокритериальной оптимизации класса $K$, в которых введено отношение предпочтения альтернатив $\rho$, важным является вопрос о внешней устойчивости множества максимальных элементов $M_{\rho}$. Напомним, что свойство внешней устойчивости подмножества $M_{\rho}$ в модели $G \in K$ задается формулой

$$
(\forall a \in A)\left(\exists a^{*} \in M_{\rho}\right) a \lesssim \rho a^{*} .
$$

Отметим, что подмножество $M_{\rho}$ всегда является внутренне устойчивым; при наличии свойства внешней устойчивости оно превращается в непустое единственное ядро отношения предпочтения $\rho$.

Дальнейшее содержание данного раздела связано с нахождением условий внешней устойчивости для моделей $G \in K$. Рассмотрим вначале модели, для которых отношением предпочтения альтернатив является Парето-предпочтение, или предпочтение по Слейтеру. Напомним, что последнее определено формулой

$$
a_{1} \leq^{S l} a_{2} \Leftrightarrow\left((\forall j \in J) q_{j}\left(a_{1}\right)<^{j} q_{j}\left(a_{2}\right)\right) \vee\left(a_{1}=a_{2}\right)
$$


Теорема 2. Пусть множество критериев $J$ конечно и все цепи $\left(\left\langle C_{j}, \leq^{j}\right\rangle\right)_{j \in J}$ удовлетворяют условию максимальности. Тогда

А) в любой модели $G \in K$, в которой отношением предпочтения альтернатив является Парето-предпочтение, множество Парето-оптимальных альтернатив будет внешне устойчивым;

В) в любой модели $G \in K$, в которой отношением предпочтения альтернатив является предпочтение по Слейтеру, множество оптимальных по Слейтеру альтернатив будет внешне устойчивым.

Лемма 1. Если все цепи $\left(\left\langle C_{j}, \leq^{j}\right\rangle\right)_{j \in J}$ удовлетворяют условию максимальности и множество J конечно, то прямое произведение $\left\langle\prod_{j \in J} C_{j}, \leq\right\rangle$ удовлетворяет условию обрьва возрастающих цуепей (условию ОВЦ).

Доказательство. Положим $|J|=n$. Предположим, что $\left\langle\prod_{j \in J} C_{j}, \leq\right\rangle$ не удовлетворяет условию ОВЦ. Тогда существует бесконечная последовательность элементов в $\prod_{j \in J} C_{j}$ вида

$$
\left(c_{1}^{1}, \ldots, c_{n}{ }^{1}\right)<\left(c_{1}^{2}, \ldots, c_{n}^{2}\right)<\ldots<\left(c_{1}^{k}, \ldots, c_{n}^{k}\right)<\ldots
$$

Из первого неравенства в (6) получаем, что существуют элементы $c_{j_{1}}^{1}<c_{j_{1}}^{2}$; из второго неравенства существуют элементы $c_{j_{2}}^{2}<c_{j_{2}}^{3}$; из $k$-го неравенства существуют элементы $c_{j_{k}}^{k}<c_{j_{k}}^{k+1}$ и т. д. Среди номеров $j_{1}, \ldots, j_{k}, \ldots$ хотя бы один из них будет повторяться бесконечное число раз, так как множество $J$ по предположению конечно, а последовательность (6) бесконечна. Пусть индекс $j_{s}$ повторяется бесконечное число раз, тогда в цепи $C_{j_{s}}$ получаем бесконечную возрастающую последовательность. Учитывая, что условие максимальности равносильно условию ОВЦ, приходим к противоречию, что завершает доказательство леммы 1.

Следствие 1. В условиях теоремы 2 всякая модель $G \in K$, в которой отношением предпочтения альтернатив является Парето-предпочтение, удовлетворяет условию (ОВЦ).

Действительно, в силу инъективности отображения $q$ (которая следует из (2)) и учитывая (3), получаем, что упорядоченное множество $\left\langle A, \omega^{P a r}\right\rangle$ изоморфно вкладывается в прямое произведение $\left\langle\prod_{j \in J} C_{j}, \leq\right\rangle$, поэтому доказанное в лемме 1 условие ОВЦ для прямого произведения влечет условие ОВЦ для упорядоченного множества $\left\langle A, \omega^{\text {Par }}\right\rangle$.

Докажем утверждение А) теоремы 2. Пусть $G$ - любая модель класса $K$, в которой в качестве отношения предпочтения альтернатив выступает предпочтение по Парето. Обозначим через $A^{*}$ множество альтернатив из $A$ максимальных относительно порядка $\leq^{P a r}$ (т. е. Парето-оптимальных альтернатив). Условие внешней устойчивости множества $A^{*}$ в такой модели согласно (5) состоит в выполнении следующей формулы:

$$
(\forall a \in A)\left(\exists a^{*} \in A^{*}\right) a \leq^{\text {Par }} a^{*} .
$$

Покажем условие (7) для модели $G$. Зафиксируем $a \in A$. Возможны два случая: 1) $a \in A^{*}$, $2) a \notin A^{*}$. В первом случае имеет место $a \leq{ }^{P a r} a$ и (7) выполнено тривиальным образом. Во втором случае из определения максимального элемента получаем, что $a<<^{P a r} a_{1}$ для некоторого $a_{1} \in A$. Если $a_{1}$ - максимальный элемент, то (7) выполнено; в противном случае из определения максимального элемента получаем, что $a_{1}<^{P a r} a_{2}$ для некоторого $a_{2} \in A$ и т. д. В результате получаем последовательность

$$
a<^{\text {Par }} a_{1}<^{\text {Par }} a_{2}<^{\text {Par }} \ldots
$$

а так как по следствию 1 упорядоченное множество $\left\langle A, \omega^{P a r}\right\rangle$ удовлетворяет условию ОВЦ, то эта последовательность оборвется на конечном номере $k$, причем имеет место $a \leq \leq^{P a r} a_{k}$ и $a_{k} \in A^{*}$, т. е. для модели $G$ выполнено условие внешней устойчивости множества Парето-оптимальных альтернатив.

Докажем теперь утверждение В) теоремы 2. Пусть $G$ - любая модель класса $K$, в которой в качестве отношения предпочтения альтернатив выступает предпочтение по Слейтеру. Обозначим 
через $A^{+}$множество тех альтернатив из $A$, которые максимальны по Слейтеру. Условие внешней устойчивости множества $A^{+}$в такой модели состоит в выполнении следующей формулы:

$$
(\forall a \in A)\left(\exists a^{+} \in A^{+}\right) a \leq^{S l} a^{+} .
$$

Покажем (8) для модели $G$. Зафиксируем $a \in A$. Возможны два случая: 1) $\left.a \in A^{+}, 2\right) a \notin A^{+}$. В первом случае имеет место $a \leq \leq^{S l} a$ и (8) выполнено тривиальным образом. Во втором случае из определения максимального элемента получаем, что $a<^{S l} a_{1}$ для некоторого $a_{1} \in A$. По уже доказанному утверждению А) для элемента $a_{1}$ найдется оптимальный, по Парето, элемент $a_{2} \in A^{*}$ такой, что $a_{1} \leq^{\text {Par }} a_{2}$. Из соотношений $a<^{S l} a_{1}$ и $a_{1} \leq^{\text {Par }} a_{2}$ следует $a<^{S l} a_{2}$. Учитывая, что паретовский оптимум содержится в слейторовском оптимуме (т. е. $A^{*} \subseteq A^{+}$), получаем $a_{2} \in A^{+}$, что заканчивает доказательство утверждения В) и теоремы 2.

Рассмотрим теперь модели класса $K$, в которых множество критериев частично упорядочено по важности отношением строгого порядка «<», а отношение предпочтения на множестве альтернатив есть отношение $\omega$, определенное формулой (4).

Теорема 3. Пусть множество критериев $J$ конечно. Для того итобы для любой модели $G \in K$ множество $M_{\omega}$ максимальных әлементов относительно порядка $\omega$ было внешне устойчивым, достаточно, итобы все цепи $\left\langle C_{j}, \leq^{j}\right\rangle_{j \in J}$ удовлетворяли условию максимальности.

Для доказательства теоремы 3 введем на множестве $\prod_{j \in J} C_{j}$ отношение порядка $\sigma$ по формуле

$$
\left(c_{j}{ }^{1}\right)_{j \in J} \leq^{\sigma}\left(c_{j}{ }^{2}\right)_{j \in J} \Leftrightarrow \forall j \in J\left(c_{j}{ }^{1} \leq^{j}{c_{j}}^{2} \vee(\exists i<j) c_{i}{ }^{1}<^{i} c_{i}{ }^{2}\right) .
$$

Тогда соответствующее отношение строгого порядка выражается в виде

$$
\left(c_{j}{ }^{1}\right)_{j \in J}<^{\sigma}\left(c_{j}{ }^{2}\right)_{j \in J} \Leftrightarrow\left(c_{j}{ }^{1}\right)_{j \in J} \leq^{\sigma}\left(c_{j}{ }^{2}\right)_{j \in J} \wedge(\exists i \in J) c_{i}{ }^{1}<^{i} c_{i}{ }^{2} .
$$

Лемма 2. Пусть множество $J$ конечно, и все цепи $\left(C_{j}\right)_{j \in J}$ удовлетворяют условию максимальности. Тогда всякая строго возрастающая цепь в упорядоченном множестве $\left\langle\prod_{j \in J} C_{j}, \leq^{\sigma}\right\rangle$ удовлетворяет условию обрьва возрастающих изепей.

Доказательство. Положим $|J|=n$. Предположим противное, т. е. что существует бесконечно возрастающая цепь вида

$$
\left(c_{1}^{1}, \ldots, c_{n}^{1}\right)<^{\sigma}\left(c_{1}^{2}, \ldots, c_{n}^{2}\right)<^{\sigma}\left(c_{1}^{3}, \ldots, c_{n}^{3}\right)<^{\sigma} \ldots .
$$

Рассмотрим первое неравенство $\left(c_{1}^{1}, \ldots, c_{n}^{1}\right)<^{\sigma}\left(c_{1}^{2}, \ldots, c_{n}^{2}\right)$. Согласно (10) $\left(\exists j_{1}\right) c_{j_{1}}^{1}<c_{j_{1}}^{2}$. Аналогично получаем $\left(\exists j_{2}\right) c_{j_{2}}^{2}<c_{j_{2}}^{3}, \ldots,\left(\exists j_{k}\right) c_{j_{k}}^{k}<c_{j_{k}}^{k+1}, \ldots$ Таким образом, имеется бесконечное число таких неравенств, а так как множество $J$ конечно, то существует индекс $j^{*} \in J$, для которого указанные неравенства выполняются бесконечное число раз:

$$
c_{j^{*}}^{k_{1}}<c_{j^{*}}^{k_{1}+1}, \quad c_{j^{*}}^{k_{2}}<c_{j^{*}}^{k_{2}+1}, \quad c_{j^{*}}^{k_{3}}<c_{j^{*}}^{k_{3}+1}, \quad \ldots .
$$

Обозначим через $N_{j^{*}}^{+}$бесконечное подмножество номеров $k \in N$, для которых выполняется строгое неравенство $c_{j^{*}}^{k}<c_{j^{*}}^{k+1}$, через $N_{j^{*}}^{0}-$ множество номеров $k \in N$ для которых выполняется равенство $c_{j^{*}}^{k}=c_{j^{*}}^{k+1}$, через $N^{-}{ }_{j^{*}}-$ множество тех номеров $k \in N$, для которых выполняется $c_{j^{*}}^{k}>c_{j^{*}}^{k+1}$ соответственно. Предположим, что множество $N_{j^{*}}^{-}$конечно. Тогда, начиная с некоторого номера $k^{*} \in N$ для всех $k>k^{*}$ выполняется неравенство $c_{j^{*}}^{k} \leq c_{j^{*}}^{k+1}$, причем, так как $N_{j^{*}}^{+}$бесконечно, строгие неравенства будут выполняться бесконечное число раз. Таким образом, в цепи $C_{j *}$ существует бесконечно возрастающая последовательность, что противоречит условию максимальности для этой цепи. Следовательно, $N_{j^{*}}^{-}$бесконечно.

Для $k \in N_{j^{*}}^{-}$неравенство $c_{j^{*}}^{k} \leq c_{j^{*}}^{k+1}$ не выполняется. Согласно определению порядка $\sigma$ при $j=j^{*}$ и $k \in N_{j^{*}}^{-}$в правой части (9) будет выполнен второй член дизъюнкции. Поэтому для любого $k \in N_{j^{*}}^{-}$найдется номер $i<j^{*}$, для которого выполнены неравенства $c_{i}^{k}<c_{i}^{k+1}$. В силу того что таких неравенств бесконечное число, а номеров $i<j^{*}$ лишь конечное число, найдется номер $j_{2}{ }^{*}<j^{*}$, для которого 
неравенства вида $c_{j_{2} *}^{k}<c_{j_{2} *}^{k+1}$ выполняются бесконечное число раз, т. е. подмножество $N_{j_{2} *}^{+}$бесконечно. Повторяя это рассуждение, получаем последовательность индексов $j^{*}=j_{1}{ }^{*}>j_{2}{ }^{*}>j_{3}{ }^{*}>\ldots$, которая в силу конечности множества $J$ должна оборваться на некотором минимальном (относительно порядка $<$ ) номере $j^{0} \in J$, для которого множество $N_{j^{0}}^{-}$будет конечным, а это влечет (как показано выше) наличие бесконечно возрастающей последовательность в цепи $C_{j}$, что противоречит условию максимальности для цепи $C_{j}$.

Из леммы 2 вытекает

Следствие 2. Пусть множество критериев $J$ конечно и все цепи $\left\langle C_{j}, \leq^{j}\right\rangle_{j \in J}$ удовлетворяют условию максимальности. Тогда всякая модель $G \in K$, в которой отношение предпочтения альтернатив определяется формулой (4), удовлетворяет условию (ОВЦ).

Доказательство теоремы 3 проводится аналогично теореме 2 с использованием следствия 2 вместо следствия 1.

\section{2. ЗАДАНИЕ ПРАВИЛА ПРЕДПОЧТЕНИЙ С ПОМОЩЬЮ ПСЕВДОФИЛЬТРА}

Рассмотрим теперь дополнительную информацию о важности локальных критериев качества в следующей форме: должно быть задано семейство $W$ важнейших групп критериев, которых, используя теоретико-игровую терминологию, будем называть выцгрывающими коалищиями критериев.

На семейство $W$ накладываются следующие условия [3]:

(PF1) нenycmoma: $W \neq \emptyset$;

(PF2) мажорантная стабильность: $S \in W, T \supseteq S \Rightarrow T \in W$;

(РF3) антидополнительность: $S \in W \Rightarrow S^{\prime} \notin W$.

Произвольное семейство $W$ подмножеств множества $J$, удовлетворяющее аксиомам (PF1)-(PF3), называется псевдофильтром над множеством $J$. Понятие псевдофильтра является обобщением понятия фильтра, играющего важную роль в некоторых вопросах алгебры, логики и топологии. В данной работе псевдофильтры используются для задания правила предпочтений. Для записи этого правила и исследования его свойств удобно с каждой моделью $G$ вида (1) связать модель $G_{Q}$ следующего вида:

$$
G_{Q}=\left\langle A,\left(\sigma_{j}\right)_{j \in J}\right\rangle
$$

где $\sigma_{j}$ есть отношение линейного квазипорядка на $A$, определенное формулой

$$
a \leq^{\sigma_{j}} a^{\prime} \Leftrightarrow q_{j}(a) \leq^{j} q_{j}\left(a^{\prime}\right)
$$

Модели, принадлежащие введенному выше классу $K$, будем записывать в виде (11).

Под решающим правилом для задания предпочтений в некотором классе моделей понимается отображение $R$, которое каждой модели этого класса ставит в соответствие рефлексивное бинарное отношение предпочтения $\rho$ определенное на базисном множестве альтернатив этой модели.

Пусть $W$ - произвольный псевдофильтр над множеством критериев $J$. Рассмотрим решающее правило $R_{W}$, которое каждой модели $G_{Q}=\left\langle A,\left(\sigma_{j}\right)_{j \in J}\right\rangle$ класса $K$ ставит в соответствие отношение предпочтения $R_{W}\left(G_{Q}\right)=\rho_{W}$ на множестве $A$, определенное следующим образом:

$$
a_{1} \lesssim^{\rho_{W}} a_{2} \Leftrightarrow\left\{j \in J: q_{j}\left(a_{1}\right) \leq^{j} q_{j}\left(a_{2}\right)\right\} \in W .
$$

Замечание. В случае, когда $W=\{J\}$, решающее правило $R_{W}$ приводит к Парето-предпочтению; если взять в качестве $W$ семейство подмножеств $S \subseteq J$, содержащих более половины элементов множества $J$, правило $R_{W}$ приводит к мажоритарному предпочтению.

Наша следующая задача - дать аксиоматическую характеристику решающего правила $R_{W}$. С этой целью введем следующие аксиомы для произвольного решающего правила $R$.

(A1) Рассмотрим две модели $G_{Q}=\left\langle A,\left(\sigma_{j}\right)_{j \in J}\right\rangle$ и $G_{Q}^{1}=\left\langle B,\left(\sigma_{j}^{1}\right)_{j \in J}\right\rangle$ класса $K$. Положим $R\left(G_{Q}\right)=\rho$, $R\left(G_{Q}^{1}\right)=\rho^{1}$. Предположим, что для элементов $a_{1}, a_{2} \in A$ и $b_{1}, b_{2} \in B$ при любом $j \in J$ выполнена следующая эквивалентность: $a_{1} \leq^{\sigma_{j}} a_{2} \Leftrightarrow b_{1} \leq^{\sigma_{j}^{1}} b_{2}$. Тогда эквивалентность $a_{1} \leq^{\rho} a_{2} \Leftrightarrow b_{1} \leq^{\rho^{1}} b_{2}$ также справедлива. 
(А2) Рассмотрим две модели $G_{Q}=\left\langle A,\left(\sigma_{j}\right)_{j \in J}\right\rangle$ и $G_{Q}^{1}=\left\langle A,\left(\sigma_{j}^{1}\right)_{j \in J}\right\rangle$ класса $K$. Зафиксируем два элемента $a_{1}, a_{2} \in A$ и предположим, что при любом $j \in J$ выполнена импликация $a_{1} \leq^{\sigma_{j}} a_{2} \Rightarrow a_{1} \leq^{\sigma_{j}^{1}} a_{2}$. Тогда условие $a_{1} \leq^{\rho} a_{2}$ влечет условие $a_{1} \leq^{\rho^{1}} a_{2}$.

(A3) Рассмотрим две модели $G_{Q}=\left\langle A,\left(\sigma_{j}\right)_{j \in J}\right\rangle$ и $G_{Q}^{1}=\left\langle A,\left(\sigma_{j}^{1}\right)_{j \in J}\right\rangle$ класса $K$. Зафиксируем два элемента $a_{1}, a_{2} \in A$ и предположим, что при любом $j \in J$ выполнена импликация $a_{1} \leq^{\sigma_{j}} a_{2} \Rightarrow a_{1}<^{\sigma_{j}^{1}} a_{2}$. Тогда условие $a_{1} \leq^{\rho} a_{2}$ влечет условие $a_{1}<^{\rho^{1}} a_{2}$.

(A4) Пусть $A-$ произвольное множество. Зафиксируем два различных элемента $a_{1}, a_{2} \in A$. Тогда найдутся такие модели $G_{Q}=\left\langle A,\left(\sigma_{j}\right)_{j \in J}\right\rangle$ и $G_{Q}^{1}=\left\langle A,\left(\sigma_{j}^{1}\right)_{j \in J}\right\rangle$ класса $K$, что для первой модели выполнено $a_{1} \leq^{\rho} a_{2}$, а для второй $\neg\left(a_{1} \leq^{\rho^{1}} a_{2}\right)$.

Теорема 4. Пусть $W-$ псевдофильтр над множеством критериев J. Правило предпочтений $R_{W}$, определенное формулой (12), удовлетворяет аксиомам (A1)-(A4).

Доказательство теоремы 4 проводится прямой проверкой. Проверим, например, выполнение аксиомы (А1).

Рассмотрим две модели $G_{Q}=\left\langle A,\left(\sigma_{j}\right)_{j \in J}\right\rangle$ и $G_{Q}^{1}=\left\langle B,\left(\sigma_{j}^{1}\right)_{j \in J}\right\rangle$ класса $K$. Положим $R_{W}\left(G_{Q}\right)=$ $=\rho_{W}, R_{W}\left(G_{Q}^{1}\right)=\rho_{W}^{1}$. Предположим, что для элементов $a_{1}, a_{2} \in A$ и $b_{1}, b_{2} \in B$ при любом æ $\in J$ выполнена эквивалентность: $a_{1} \leq{ }^{\sigma_{j}} a_{2} \Leftrightarrow b_{1} \leq \sigma_{j}^{1} b_{2}$.

Тогда $\left\{j \in J: a_{1} \leq^{\sigma_{j}} a_{2}\right\}=\left\{j \in J: b_{1} \leq^{\sigma_{j}^{1}} b_{2}\right\}$, следовательно, условия $\left\{j \in J: a_{1} \leq^{\sigma_{j}} a_{2}\right\} \in W$ и $\left\{j \in J: b_{1} \leq^{\sigma_{j}^{1}} b_{2}\right\} \in W$ равносильны, поэтому высказывания $a_{1} \leq^{\rho_{W}} a_{2}$ и $b_{1} \leq^{\rho_{W}^{1}} b_{2}$ эквивалентны между собой.

Установим теперь обращение теоремы 4.

Теорема 5. Зафиксируем семейство шкал $\left(C_{j}, \leq_{j}\right)_{j \in J}$ для измерения качественных критериев. Пусть $R-$ правило задания предпочтений, которое каждой модели $G_{Q}=\left\langle A,\left(\sigma_{j}\right)_{j \in J}\right\rangle$ класса K ставит в соответствие рефлексивное бинарное отношение предпочтения $R\left(G_{Q}\right)=\rho$ на множестве альтернатив $A$, причем для $R$ выполнены аксиомы (A1)-(A4). Тогда существует такой псевдофильтр $W$ над $J$, что $R=R_{W}$.

Наметим доказательство теоремы 5 . Определим семейство выигрывающих коалиций критериев $W$ следующим образом. Для произвольного подмножества $S \subseteq J$ условие $S \in W$ полагаем выполненным тогда и только тогда, когда существует модель $\bar{G}_{Q}^{1}=\left\langle\bar{A},\left(\bar{\sigma}_{j}^{1}\right)_{j \in J}\right\rangle$ класса $K$ и такие элементы $\overline{a_{1}}, \overline{a_{2}} \in \bar{A}$, что

$$
\overline{a_{1}} \leq_{\bar{\rho}}^{\overline{a_{2}}} \wedge\left\{j \in J: \overline{a_{1}} \leq^{\overline{\sigma_{j}}} \overline{a_{2}}\right\}=S
$$

(мы обозначаем $\bar{\rho}=R\left(\bar{G}_{Q}^{1}\right)$ ).

Далее определяем правило $R_{W}$ для предпочтений в классе $K$ формулой (12). Покажем, что $R_{W}=R$. Для этого достаточно убедиться, что для любой модели $G_{Q}=\left\langle A,\left(\sigma_{j}\right)_{j \in J}\right\rangle$ класса $K$ выполнена эквивалентность

$$
a_{1} \leq^{\rho} a_{2} \Leftrightarrow\left\{j \in J: a_{1} \leq^{\sigma_{j}} a_{2}\right\} \in W .
$$

В самом деле, импликация слева направо в (14) верна по определению семейства $W$. Покажем обратную импликацию. Пусть выполнена правая часть формулы (14). Это означает, что существует модель $\bar{G}_{Q}=\left\langle\bar{A},\left(\bar{\sigma}_{j}\right)_{j \in J}\right\rangle$ класса $K$ и элементы $\overline{a_{1}}, \overline{a_{2}} \in \bar{A}$, для которых выполнено

$$
\overline{a_{1}} \leq^{\bar{\rho}} \overline{a_{2}} \wedge\left\{j \in J: \overline{a_{1}} \leq^{\overline{\sigma_{j}}} \overline{a_{2}}\right\}=\left\{j \in J: a_{1} \leq^{\sigma_{j}} a_{2}\right\} .
$$

Тогда условия $a_{1} \leq^{\sigma_{j}} a_{2}$ и $\overline{a_{1}} \leq^{\overline{\sigma_{j}}} \overline{a_{2}}$ при любом $j \in J$ эквивалентны между собой; по аксиоме (A1) высказывания $a_{1} \leq^{\rho} a_{2}$ и $\overline{a_{1}} \leq^{1} \overline{a_{2}}$ будут равносильными, а так как высказывание $\overline{a_{1}} \leq^{\bar{\rho}} \overline{a_{2}}$ согласно (15) истинно, то истинным будет и высказывание $a_{1} \leq^{\rho} a_{2}$. Таким образом, формула (14) проверена и равенство $R_{W}=R$ установлено. Для завершения доказательства теоремы 5 осталось показать, что определенное формулой (13) семейство подмножеств образует псевдофильтр. Это производится простой проверкой аксиом (PF1)-(PF3). 


\title{
Библиографический список
}

1. Подиновский В. В., Ногин В. Д. Парето-оптимальные решения многокритериальных задач. М. : Наука, 1982. 256 c.

2. Смирнова Д. С. Модели многокритериальной оптимизации с частично упорядоченным множеством критериев // Компьютерные науки и информационные тех- нологии : материалы междунар. науч. конф. Саратов : Издат. центр «Наука», 2012. С. 293.

3. Розен В. В. Математические модели многокритериальной оптимизации по качественным критериям // Компьютерные науки и информационные технологии : материалы междунар. науч. конф. Саратов : Издат. центр «Наука», 2012. С. 266.

\section{Models of Multi-criteria Optimization with Quality Criteria}

\section{V. Rozen, D. S. Smirnova}

Saratov State University, Russia, 410012, Saratov, Astrahanskaya st., 83, Rozenvv@ info.sgu.ru, smirnova @okean-kv.ru

We consider mathematical models of multi-criteria optimization with quality criteria. The main problem is a construction of preference relations on the set of alternatives and an investigation of its mathematical properties. Two methods for contraction of Pareto-optimal set are proposed. The first method is based on introduction of a partial order relation on the set of criteria and the second leans selection of the most important groups of criteria.

Key words: model of multi-criteria optimization, preference relation, Pareto-optimality, winning coalition of criteria.

\section{References}

1. Podinovskiy V. V., Noghin V. D. Pareto-optimal'nye resheniia mnogokriterial'nykh zadach [Pareto-optimal decisions of multi-criteria problems]. Moscow, Nauka, 1982, 256 p. (in Russian).

2. Smirnova D. S. Modeli mnogokriterial'noi optimizatsii s chastichno uporiadochennym mnozhestvom kriteriev [Models of multi-criteria optimizations with partially ordered set of criteria]. Komp'iuternye nauki $i$ infor- matsionnye tekhnologii : materialy mezhdunar. nauch. konf., Saratov, 2012, pp. 293 (in Russian).

3. Rozen V. V. Matematicheskie modeli mnogokriterial'noi optimizatsii po kachestvennym kriteriiam [Mathematical models of multi-criteria optimization under quality criteria]. Komp'iuternye nauki $i$ informatsionnye tekhnologii : materialy mezhdunar. nauch. konf. Saratov, 2012. pp. 266 (in Russian).

УДК 519.17

\section{УПОРЯДОЧЕННОЕ МНОЖЕСТВО СВЯЗНЫХ ЧАСТЕЙ МНОГОУГОЛЬНОГО ГРАФА}

\begin{abstract}
В. Н. Салий
Кандидат сризико-математических наук, заведующий каседрой теоретических основ компьютерной безопасности и криптограсрии, профессор, Саратовский государственный университет им. Н. Г. Чернышевского, SaliiVN@ info.sgu.ru

Под многоугольным грасром понимается ориентированный графр, полученный из цикла путем некоторой ориентации его ребер. Множество абстрактных (т.е. рассматриваемых с точностью до изоморфизма) связных частей многоугольного графра упорядочивается отношением вложимости графов. Получено описание многоугольных графов, для которых это упорядоченное множество является решеткой.
\end{abstract}

Ключевые слова: многоугольный графр, линейный граф, двоичный вектор, двойственность, упорядоченное множество, решетка.

Под графом понимается пара $G=(V, \alpha)$, где $V-$ конечное непустое множество и $\alpha \subseteq V \times V-$ отношение на нем. Элементы множества $V$ называются вершинами графа, а пары, входящие в отношение смежности $\alpha,-$ дугами.

Если $V^{\prime} \subseteq V$ и $\alpha^{\prime} \subseteq \alpha$, то граф $G^{\prime}=\left(V^{\prime}, \alpha^{\prime}\right)$ называется частью графа $G$. В случае, когда $\alpha^{\prime}=\alpha \cap\left(V^{\prime} \times V^{\prime}\right)$, говорят, что $G^{\prime}$ является подграфом графа $G$.

Пусть $G=(V, \alpha)$ и $H=(U, \beta)$ - некоторые графы. Вложение графа $G$ в граф $H$ - это такое инъективное отображение $\varphi: V \rightarrow U$, что $\left(\forall v, v^{\prime} \in V\right)\left(\left(v, v^{\prime}\right) \in \alpha \Longrightarrow\left(\varphi(v), \varphi\left(v^{\prime}\right)\right) \in \beta\right)$. Если граф $G$ 